\title{
ATLAS Upgrades towards the High Luminosity LHC: extending the discovery potential
}

\section{Silvia Miglioranzi*}

Author affiliation: CERN, Aristotle Univ. of Thessaloniki (GR)

E-mail: Silvia.Miglioranzidcern.ch

ATLAS has successfully collected 5.25 and $21.7 \mathrm{fb}^{-1}$ during LHC run I at $\sqrt{s}=7$ and $8 \mathrm{TeV}$, respectively. However, the LHC planning for the years to come, with a foreseen delivered luminosity up to $3000 \mathrm{fb}^{-1}$ at $\sqrt{s}=14 \mathrm{TeV}$, is a great challenge for the detectors. ATLAS has already started a staged upgrade programme involving most of the detector subsystems, in order to exploit the full physics accessible with the expected luminosity increase. This report describes the ATLAS upgrade plans together with the expected detector performances and physics capabilities.

XXI International Workshop on Deep-Inelastic Scattering and Related Subject-DIS2013, 22-26 April 2013

Marseilles, France

${ }^{*}$ Speaker. 


\section{Introduction}

ATLAS [1] is a multi-purpose detector installed in one of the interaction regions of the CERN Large Hadron Collider (LHC). During LHC run I, ATLAS has successfully taken data at $\sqrt{s}=7$ and $8 \mathrm{TeV}$, collecting an integrated luminosity of $5.25 \mathrm{fb}^{-1}$ and $21.7 \mathrm{fb}^{-1}$, respectively. The maximum instantaneous luminosity reached by LHC at the start of fill has been $\mathscr{L}_{\text {peak }}=7.73 \times 10^{33} \mathrm{~cm}^{-2} \mathrm{~s}^{-1}$. In the next years, LHC will undergo a series of upgrades, planned in three stages (phase-0, phase-I, phase-II), changing the collider parameters for the following running periods as shown in Table 1. The average number of interactions per crossing will approximately increase from 20 to 140 .

\begin{tabular}{l|c|c|c}
\hline Upgrade Phase (run period) & $\sqrt{(s)(\mathrm{TeV})}$ & exp. $\mathscr{L}_{\text {peak }}\left(\mathrm{cm}^{-2} \mathrm{~s}^{-1}\right)$ & exp. $\mathscr{L}_{\text {integrated }}\left(\mathrm{fb}^{-1}\right)$ \\
\hline phase-0 (2015-2017) & $13-14$ & $1 \times 10^{34}$ & 100 \\
phase-I (2019-2021) & 14 & $2-3 \times 10^{34}$ & 300 \\
phase-II (2023- ) & 14 & $5-7 \times 10^{34}$ & 3000 \\
\hline
\end{tabular}

Table 1: LHC main parameters after the planned upgrades.

This will require corresponding upgrades to the ATLAS detector to maintain physics acceptance in the high luminosity and harsh background environment. The ATLAS physics program will cover the following physics topics: precise studies of the observed resonance with mass $126 \mathrm{GeV}$, studies of associated Higgs production, searches for new phenomena and new particles predicted by theoretical models such as SUSY, technicolor, compositeness, extra-dimensions. Indirect searches for new physics will also continue through precision measurements of SM processes and looking for deviations from the predictions. The upgrades of detector, trigger and computing system are planned in three long LHC technical shutdowns splitting the data taking periods.

\section{Phase-0 Upgrade}

In the running period 2015-2017, LHC will increase the center of mass energy of pp collisions to $\sqrt{s}=13-14 \mathrm{TeV}$ with a peak luminosity of $\mathscr{L}_{\text {peak }}=10^{34} \mathrm{~cm}^{-2} \mathrm{~s}^{-1}$ delivering about $100 \mathrm{fb}^{-1}$ of data. The ATLAS experiment will undergo several consolidation works in order to cope with the new running conditions: new beam pipe in the central and forward region, new evaporative cooling system for the Inner Detector, new pixel services, new Diamond Beam Monitor, new calorimeter power supplies, completion of the muon spectrometer, additional neutron shielding in the forward region. The installation of a new layer of pixels, the major upgrade of phase- 0 , is described in the next paragraph.

\subsection{Instertable B-Layer}

An additional 4th pixel layer, named Insertable B-Layer (IBL) [2], will be added between the current innermost pixel layer (B-layer) and the beam pipe, at a sensor average radius of $33 \mathrm{~mm}$ as shown in Fig.1 left. A new $25 \mathrm{~mm}$ radius Beryllium beam pipe around the interaction region will allow to accommodate the IBL. An hybrid configuration of $50 \times 250 \mu \mathrm{m}$ planar and 3D sensors will be mounted on Carbon-foam staves. Simulation studies have shown that the additional layer 

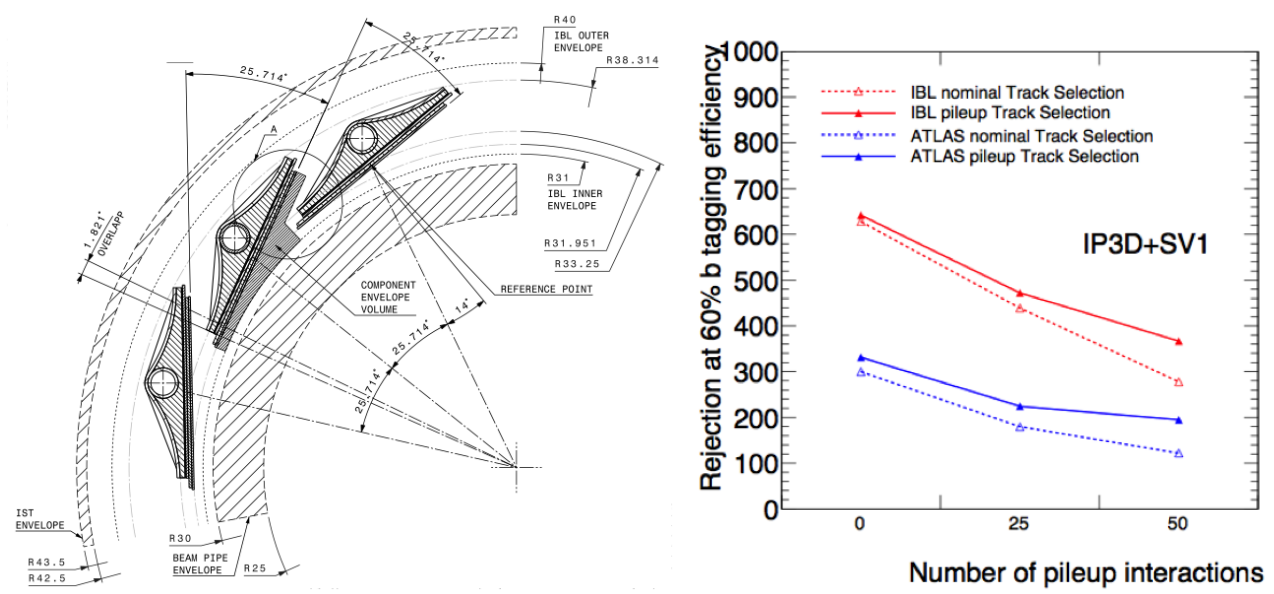

Figure 1: Left: IBL layout: $\mathrm{r} \phi$ view [2]. Right: Light jet rejection at a b-tagging efficiency of $60 \%$ versus pile-up for standard ATLAS (blue) and ATLAS+IBL (red) [2].

of pixels will provide a factor 3 increase in light jet rejection while maintaining the b-tagging efficiency (see Fig.1 right). When the B-layer will suffer from radiation damage and high pileup occupancies at high luminosity, IBL will allow to maintain good tracking performances.

\section{Phase-I Upgrade}

After phase-I upgrade LHC will reach a peak luminosity of $2-3 \times 10^{34} \mathrm{~cm}^{-2} \mathrm{~s}^{-1}$ and deliver about $300 \mathrm{fb}^{-1}$ of pp collision data. The increased statistics will allow to accurately study the Higgs-like resonance with a mass of about $126 \mathrm{GeV}$ observed in run I, and precisely measure its properties (width, branching ratios, couplings to fermions and bosons). To maximize the physics acceptance, a low $\mathrm{p}_{T}(\sim 20 \mathrm{GeV})$ single isolated lepton trigger at the first stage of the trigger (Level1) will be required. The total output bandwidth of the Level-1 is constrained to $100 \mathrm{kHz}$ with about $20 \mathrm{kHz}$ allocated to both single isolated electron and single isolated muon trigger. Handling luminosities well beyond the nominal values and controlling the increasing trigger rates while retaining low $\mathrm{p}_{T}$ lepton thresholds at Level-1 will be the main focus of the ATLAS upgrades consisting mainly in a calorimeter trigger with finer granularity at Level-1, new muon small wheels in the endcaps using micro-megas and thin gap chamber technologies and fast tracking capabilities for all Level-1 accepted triggers. Detailed ATLAS plans for phase-I are described in [3].

\subsection{Muon New Small Wheels}

To maximize the physics acceptance, a single muon trigger with a $\mathrm{p}_{T}$ threshold above 20 $\mathrm{GeV}$ at Level-1 is planned. The single muon trigger rates are dominated by fakes in the forward region originating from cavern backgrounds. Adding trigger planes in the small wheel region (i.e. before the endcap toroid) measuring the muon track vector with $1 \mathrm{mrad}$ angular precision, will significantly reduce the fake contributions that dominate in this region. The new small wheel chambers will cover the rapidity region $1.2<|\eta|<2.4$. Two quadruplets of TGCs for triggering purposes will surround two quadruplets of micromegas chambers for high precision tracking. The 


\begin{tabular}{c|c|c}
\hline Level-1 trigger $(\mathrm{GeV})$ & Efficiency $(\%)$ & Rate $(\mathrm{kHz})$ \\
\hline $\mathrm{p}_{T}(\mu)>20$ & 82 & 40 \\
$\mathrm{p}_{T}(\mu)>40$ & 50 & 15 \\
$\mathrm{p}_{T}(\mu)>20$ with NSW & 78 & 18 \\
\hline
\end{tabular}

Table 2: Efficiencies and rates of the level-1 trigger for the WH associated production process [3].

addition of the new small wheel will reduce by a factor 3 the fake rate contributions allowing the deployment of lower muon $\mathrm{p}_{T}$ thresholds at Level- 1 . This will significantly increase the acceptance for the associated production of Higgs as shown in Table 2.

\subsection{Fast Track Trigger}

The Fast Track Trigger is a highly parallel hardware system performing the track finding and fitting for all Level-1 accepted triggers and providing the track parameters at the beginning of the Level-2 processing. The hits in the silicon detectors are matched to $10^{9}$ patterns pre-stored in associative memories. Then a fast linear fitting is performed on FPGAs for matched tracks using the hit information in the silicon detectors yielding precise track information.

\subsection{Higher Granularity Level-1 Calorimeter Signals}

The current Level-1 EM calorimeter trigger applies $\mathrm{E}_{T}$ thresholds on trigger towers with size $\Delta \phi \times \Delta \eta=0.1 \times 0.1$. A powerful discrimination of jets from electrons can be obtained using a finer segmentation $(\Delta \phi \times \Delta \eta=0.025 \times 0.1)$ in the second sampling layer where the EM shower reaches its maximum. Applying the new shower shape cuts provided by the upgraded readout a factor 3 rejection can be obtained.

\section{Phase-II Upgrade}

In its ultimate running conditions LHC is expected to reach a peak luminosity of $5-7 \times 10^{34}$ $\mathrm{cm}^{-2} \mathrm{~s}^{-1}$ (depending on the leveling option). The expected delivered luminosity at $\sqrt{s}=14 \mathrm{TeV}$ of about $3000 \mathrm{fb}^{-1}$ will further increase the physics reach of the ATLAS experiment. Precision measurements of the Higgs boson, reducing uncertainties on $\sigma \times B R$ to less than 5\% for some Higgs decay modes, will be possible. The ratio of the partial widths provides a measurement of the Higgs couplings, its precision measurement will offer an indirect probe of new physics at a 5-15 $\%$ level. The high rates and increased radiation fluences expected at the HL-LHC, together with the aging of the current detectors and readout electronics, will require a significant upgrade of the ATLAS detector systems. The main phase-II upgrade of the ATLAS detector are reported below, while a detailed description can be found in [4].

\subsection{New Inner Detector}

The current inner tracker has been designed to operate at the LHC nominal parameters. At HL-LHC it will need therefore to be replaced to cope with the performance requirements. The new tracker, (layout in Fig. 2), will be an all-silicon detector with pixels layers in the innermost part, 


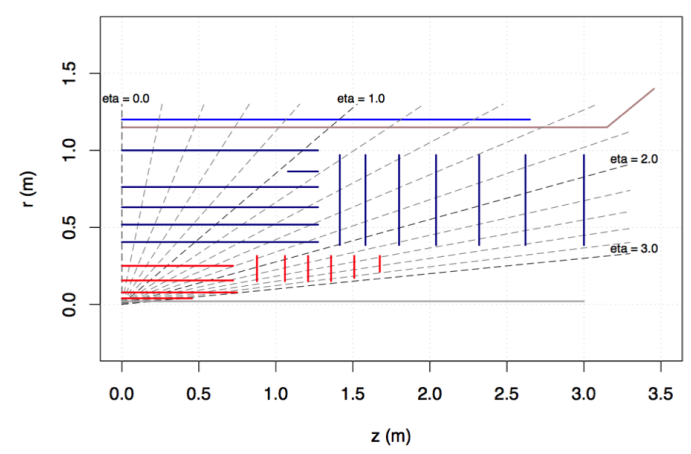

Figure 2: Layout of the new all-silicon Inner Tracker [4].

surrounded by microstrip sensors. It will cover a pseudorapidity range up to $|\eta|<2.5$ (pixels up to $|\eta|<2.7)$ providing at least 14 hits for each track.

\subsection{TDAQ Upgrade}

The planned trigger upgrades for phase-II foresee full granularity of calorimeter and the introduction of inner tracker information at Level-1. The single lepton trigger rate will be reduced by combining the inner tracker info with the muon and calorimeter triggers. The $\tau$-identification and the b-tagging will greatly benefit from the precise tracking info. The introduced complexity will need longer processing time: the Level-1 latency will be increased from 2.5 to $20 \mu$ s and the present single-stage Level-1 trigger will evolve into a 2-level scheme, with a maximum accepted rate of $500 \mathrm{kHZ}$ at Level-0 ( $5 \mu$ s latency) and $200 \mathrm{kHz}$ at Level-1 (20 $\mu$ s latency). The last stage of the trigger, High Level Trigger (HLT), will benefit from the increased computing power available through the progress in computing technology and new software will be developed to exploit the enhanced performance.

\section{Conclusions}

The ATLAS upgrade program has been planned to allow the full exploitation of the physics potential of LHC up to its ultimate luminosity. From phase-0 through phase-II the proposed upgrades should be able to preserve the detector performances while operating in harsher background conditions.

\section{References}

[1] ATLAS Collaboration, The ATLAS Experiment at the CERN Large Hadron Collider, JINST 3, S08003 (2008)

[2] ATLAS collaboration, Insertable B-Layer, Technical Design Report, CERN/LHCC-2010-013

[3] ATLAS collaboration, Letter of Intent for the Phase-I Upgrade of the ATLAS Experiment, CERN/LHCC-2011-012, LHCC-I-020

[4] ATLAS Collaboration, Letter of Intent for the Phase-II Upgrade of the ATLAS Experiment, CERN/LHCC-2012-022 